\title{
JUCYS-MURPHY ELEMENT AND WALKS ON MODIFIED YOUNG GRAPH
}

\author{
AKIHITO HORA \\ Faculty of Environmental Science and Technology, Okayama University \\ Tsushima, 700-8530 Okayama, Japan \\ E-mail: hora@ems.okayama-u.ac.jp
}

Dedicated to Professor Yasuo Yamasaki for his 70th birthday

\begin{abstract}
Biane found out that irreducible decomposition of some representations of the symmetric group admits concentration at specific isotypic components in an appropriate large $n$ scaling limit. This deepened the result on the limit shape of Young diagrams due to Vershik-Kerov and Logan-Shepp in a wider framework. In particular, it is remarkable that asymptotic behavior of the Littlewood-Richardson coefficients in this regime was characterized in terms of an operation in free probability of Voiculescu. These phenomena are well understood through highest order analysis in the Kerov-Olshanski algebra of polynomial functions on Young diagrams with respect to the weight degree. Taking this point of view of highest order analysis into account, we show an asymptotic formula for moments of the Jucys-Murphy element by considering an appropriate graph structure on the Young diagrams which parametrize the conjugacy classes.
\end{abstract}

1. Introduction. This note grew out of an effort to understand Biane's works [1] and [2] where a remarkable connection of asymptotic theory for representations of the symmetric groups with free probability of Voiculescu is revealed. In particular, an asymptotic behavior (or a certain thermodynamical limit) of the Littlewood-Richardson coefficients is described by using free convolution. One of our motivations is to give an alternative proof of this important result within the framework of representations of the symmetric groups, namely without appealing to those of the general linear groups.

Asymptotic theory for representations of the symmetric groups, which was initiated by Vershik, Kerov, Olshanski et al., has enjoyed rapid development in connection with a wide

2000 Mathematics Subject Classification: Primary 20P05; Secondary 20C30, 05E10.

Key words and phrases: symmetric group, Jucys-Murphy element, Young diagram, noncrossing partition.

Research supported by JSPS grant 16540154.

The paper is in final form and no version of it will be published elsewhere. 
variety of topics in harmonic analysis and probability theory, including random matrices, point processes, free probability, and representations of the infinite symmetric group. We refer to Kerov [10] and Vershik [17] which outline development of the theory. Concerned with behavior of objects related to representations of the symmetric group $S(n)$ as $n \rightarrow$ $\infty$, we can observe various phenomena according to the regimes of scaling limits. The set $\mathbb{Y}_{n}$ of the Young diagrams with $n$ boxes parametrizes both the equivalence classes of the irreducible representations and the conjugacy classes of $S(n)$. The Plancherel measure is introduced on $\mathbb{Y}_{n}$ in several canonical manners, such as the Plancherel theorem in Fourier analysis (or irreducible decomposition of the regular representation), the distribution of a canonical Markov chain on the Young graph, and the push-forward of the uniform probability on $S(n)$ by the Robinson-Schensted correspondence. When diagrams in $\mathbb{Y}_{n}$ are rescaled by $1 / \sqrt{n}$ horizontally and vertically and distributed according to the Plancherel measure, the famous limit shape appears as a typical diagram in $n \rightarrow \infty$ limit, as shown by Vershik-Kerov [18] and Logan-Shepp [12]. This result was a starting point of subsequent great progress.

A fantastic idea due to Vershik-Kerov, which enables us to treat scaling limits well, is to embed Young diagrams into a function space and further a space of measures on $\mathbb{R}$. The notion of transition measure for a diagram due to Kerov is of particular use. Based on a series of Kerov's works, Biane found in [1] that free probability theory describes properly the asymptotic behavior of representations of the symmetric groups in the same scaling regime as the limit shape is observed. Biane showed the following results. In this regime, the irreducible character of $S(n)$ enjoys an asymptotic formula as $n \rightarrow \infty$ in terms of free cumulants of the transition measure corresponding to the irreducible representation. Also in the same regime as $n \rightarrow \infty$, several representations admit a concentration phenomenon in which certain dominant isotypic components can be specified in the irreducible decompositions. Moreover, the specific components are determined from the original representations through appropriate operations in free probability theory. In particular, the apperance of the limit shape due to Vershik-Kerov and Logan-Shepp is this concentration in irreducible decomposition of the regular representation of $S(n)$. Here the transition measure of the limit shape is the standard semi-circle distribution of Wigner. In these works of Biane, a central role is played by the Jucys-Murphy element

$$
J_{n}=(1 n+1)+(2 n+1)+\cdots+(n n+1) \in \mathbb{C}[S(n+1)]
$$

which commutes with $S(n)$ and fully describes the branching rule of the restriction of each irreducible representation of $S(n+1)$ to $S(n)$. Throughout this note, $S(n)$ is embedded as the stabilizer of the letter $n+1$ in $S(n+1)$.

Let us assemble some notations in symmetric groups and Young diagrams. $\mathbb{Y}$ denotes the set of Young diagrams. For $\lambda \in \mathbb{Y}$, the number of boxes, rows and columns are denoted by $|\lambda|, \operatorname{row}(\lambda)$ and $\operatorname{col}(\lambda)$ respectively. The number of $j$-rows of $\lambda$ is denoted by $m_{j}(\lambda)$. Set $\overline{\mathbb{Y}}=\left\{\lambda \in \mathbb{Y} \mid m_{1}(\lambda)=0\right\}$ and $\overline{\mathbb{Y}}_{k}=\{\lambda \in \overline{\mathbb{Y}}|| \lambda \mid=k\}$. For $\rho, \sigma \in \mathbb{Y}$, their union $\rho \cup \sigma$ is defined by $m_{j}(\rho \cup \sigma)=m_{j}(\rho)+m_{j}(\sigma)$. We use also the cycle notation $\rho=\left(1^{m_{1}(\rho)} 2^{m_{2}(\rho)} \ldots j^{m_{j}(\rho)} \ldots\right)$ and the row coordinates $\rho=\left(\rho_{1} \geq \rho_{2} \geq \cdots \geq \rho_{i} \geq \cdots\right)$. The conjugacy class of $S(n)$ and the adjacency operator corresponding to $\rho \in \overline{\mathbb{Y}},|\rho| \leq n$ 


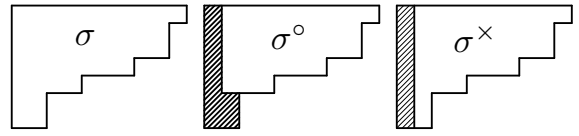

Fig. 1. $\sigma^{\circ}$ and $\sigma^{\times}$

are denoted by $C_{\rho \cup\left(1^{n-|\rho|}\right)}$ and $A_{\rho \cup\left(1^{n-|\rho|}\right)}=\sum_{x \in C_{\rho \cup\left(1^{n-|\rho|}\right)}} x$ respectively. Let $l(g)$ be the distance between $g \in S(n)$ and the unit element $e$ (or the length of $g$ ) on the Cayley graph of $S(n)$ where all transpositions are taken as generators. $l$ is a class function on $S(n)$. If $g \in C_{\rho \cup\left(1^{n-|\rho|}\right)}$, we have $l(g)=n-\sharp\{$ cycles in $g\}=|\rho|-\operatorname{row}(\rho)$. We then define the length function $l$ on $\overline{\mathbb{Y}}$ by $l(\rho)=|\rho|-\operatorname{row}(\rho)$. $\sigma^{\circ}$ denotes the diagram made of $\sigma \in \overline{\mathbb{Y}}$ by removing all 2-rows and the 1st column, while $\sigma^{\times}$the one by removing just the 1 st column (Figure 1). For $\sigma \in \mathbb{Y}_{k}$, let $N C(\sigma)$ denote the set of all noncrossing partitions of $\{1,2, \ldots, k\}$ with $\sigma$ as their block structure (namely, having $m_{j}(\sigma)$ blocks of length $j$ ). Conditional expectation $\mathbb{E}_{n}: \mathbb{C}[S(n+1)] \longrightarrow \mathbb{C}[S(n)]$ is defined to fix the elements in $S(n)$ and to map $S(n+1) \backslash S(n)$ to 0 .

Here is the main result of this note.

THEOREM 1. The following asymptotic expression holds for $k \in \mathbb{N}=\{1,2, \ldots\}$ :

$$
\mathbb{E}_{n} J_{n}^{k}=\sum_{\sigma \in \overline{\mathbb{Y}}_{k}}|N C(\sigma)| n^{l(\sigma)}\left(1+O\left(n^{-1}\right)\right) \frac{A_{\sigma^{\circ} \cup\left(1^{n-\left|\sigma^{\circ}\right|}\right)}}{\left|C_{\sigma^{\circ} \cup\left(1^{n-\left|\sigma^{\circ}\right|}\right)}\right|} .
$$

Note the advantage that the range of sum depends only on $k$ and not on $n$. The meaning of Theorem 1 will be clearer when we put Eq. (1) into the conjugacy class algebra (or, applying irreducible characters, into the polynomial functions on $\mathbb{Y}$ ) and consider the weight degree. Each summand in the right hand side of Eq. (1) is expressed as

$$
|N C(\sigma)| a_{\sigma^{\times} ; n}+\text { (lower terms) }
$$

with respect to the weight degree. See $\S 4$ for necessary definitions. The weight degree of $a_{\sigma^{\times} ; n}$ is equal to $\left|\sigma^{\times}\right|+\operatorname{row}(\sigma)=|\sigma|=k$.

As a continuation of the works of Kerov and Biane along this direction, some recent works of Śniady are remarkable. In [13] and [14], Śniady studied not only analysis of the highest degree terms but also transformation rules involving lower degree terms. As an application, he obtained a universal feature of the fluctuation of Young diagrams.

The aim of this note is more modest. In $\S 2$ we give an elementary proof of Theorem 1 by introducing random walk on Young graph $\overline{\mathbb{Y}}$ which is stratified in a noncanonical way. In $\S 3$ we summarize discussions about how the concentration phenomenon is observed in irreducible decomposition by applying Theorem 1. Asymptotic theory for symmetric groups has an important feature as permutation models for free probability. It is therefore meaningful to describe how operations in free probability come out in the scaling limits by way of random permutation methods which are logically independent of random matrix models. The main theorem and its proof are quite elementary and need no preliminaries. For convenience of readers, we collect in $\S 4$ some notions and terminology which may not be well-known and are necessary for understanding the discussion on the concentration phenomenon in $\S 3$ (as well as some parts in this Introduction). 


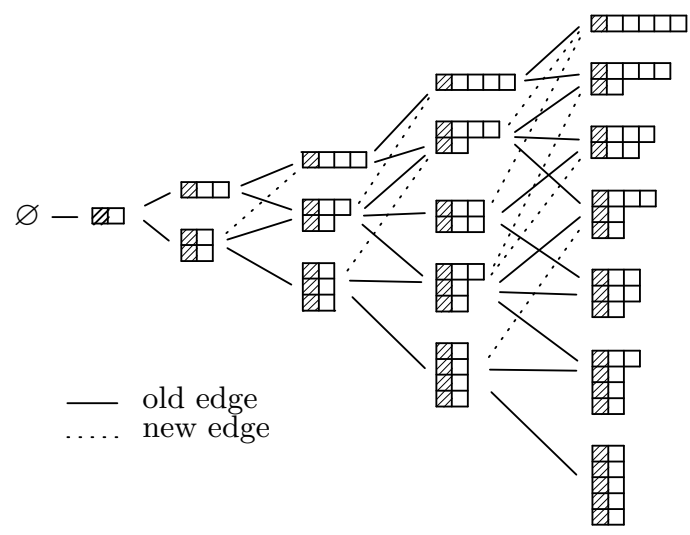

Fig. 2. Stratification of $\overline{\mathbb{Y}}$ and its graph structure

\section{Proof of Theorem 1}

2.1. Suppose $n \gg k$. In the expression

$$
J_{n}^{k}=\sum_{i_{1}, \ldots, i_{k} \in\{1, \ldots, n\}}\left(i_{k} *\right) \cdots\left(i_{1} *\right) \quad \text { where } *=n+1,
$$

each term induces a $k$-walk starting at $e$ on the Cayley graph of $S(n+1)$ as well as its associated 'shadow walk' on $\overline{\mathbb{Y}}$. Here $\overline{\mathbb{Y}}$ is considered to parametrize conjugacy classes and is given the stratification according to length function $l$ (Figure 2). One step transition increases or decreases the length just by 1 and hence causes an up or down among the strata. This stratification was used also in [4] to introduce appropriate creation and annihilation operators. Acted on by $\mathbb{E}_{n}$, a term really contributes to the sum only if $\left(i_{k} *\right) \cdots\left(i_{1} *\right) \in S(n)$. Its type is characterized by the following. (In [3], those partitions determined by such indices $i_{1}, \ldots, i_{k}$ are said to be admissible.) The proof is given in the next subsection.

Lemma 1. Let $\rho \in \overline{\mathbb{Y}}$ be given. The following are equivalent.

(i) There exist $i_{1}, \ldots, i_{k} \in\{1, \ldots, n\}$ such that $\left(i_{k} *\right) \cdots\left(i_{1} *\right)$ is of $\rho$-type and in $S(n)$.

(ii) $l(\rho)$ has the same parity with $k$ and satisfies $|\rho|+\operatorname{row}(\rho) \leq k$.

(iii) $\rho=\sigma^{\circ}$ holds for some $\sigma \in \overline{\mathbb{Y}}_{k}$.

Lemma 1 yields

$$
\mathbb{E}_{n} J_{n}^{k}=\sum_{\sigma \in \overline{\mathbb{Y}}_{k}} \sum_{g \in C_{\sigma^{\circ} \cup\left(1^{n-\left|\sigma^{\circ}\right|}\right)}}\left(\mathbb{E}_{n} J_{n}^{k}\right)(g) g .
$$

For each $\sigma \in \overline{\mathbb{Y}}_{k}$, we count up the second sum in Eq. (2) asymptotically (neglecting lower orders). To describe possible ups and downs caused by multiplying $(i *)$ from the left, we introduce the edges for $\overline{\mathbb{Y}}$ as Figure 2. We have the stratification of $\overline{\mathbb{Y}}$ with respect to $l$ by pasting a copy of the first column at every diagram in the usual Young graph $\mathbb{Y}$. In Figure 2, $\overline{\mathbb{Y}}$ inherits the old edges from $\mathbb{Y}$ in this operation, while the new edges indicate that the two diagrams are communicated each other by joining two rows or by dividing one row. We classify the contributing terms in the second sum of Eq. (2) according to the 
associated $k$-walks on the modified Young graph $\overline{\mathbb{Y}}$ in an asymptotic manner. If an up step is caused by multiplying $(i *)$, we can assume that the letter $i$ has never appeared since otherwise the contributions are of lower orders. (For example, we neglect the case of going up along a new edge.) Clearly $*$ must jump out of a nontrivial cycle in the final step. The associated (non-Markovian) random walk on $\overline{\mathbb{Y}}$ is governed by the following rule.

1. The final step is going down along an old edge.

2. Suppose you are now at $\rho \in \overline{\mathbb{Y}}$. Your next diagram is chosen by:

(a) if you came up to $\rho$ along an old edge

i. add a box to the row just lengthened [up]

ii. divide the row just lengthened [down]

(b) if you came down to $\rho$ along an old edge

i. add a 2-row [up]

ii. add a box to the row just shortened [up]

iii. divide the row just shortened [down]

(c) if you came down to $\rho$ along a new edge

i. add a box to either row just divided [up]

ii. divide either row just divided [down].

Given a sample of $k$-walk obeying this rule, we can specify the row containing $*$ (up to the ones of equal length) at every step by tracing backward the walk to $\varnothing$.

For $\sigma \in \overline{\mathbb{Y}}_{k}$, let $W(\sigma)$ denote the $k$-walks from $\varnothing$ to $\sigma^{\circ}$ obeying this rule. If a $k$-walk $w \in W(\sigma)$ has $u$ up steps, the number of corresponding paths on the Cayley graph of $S(n+1)$ to $w$ is $n^{u}$ up to lower orders. Since $u$ is given by

$$
u=\frac{k+l\left(\sigma^{\circ}\right)}{2}=\frac{|\sigma|+l\left(\sigma^{\circ}\right)}{2}=|\sigma|-\operatorname{row}(\sigma)=l(\sigma)
$$

from Eq. (5), we have

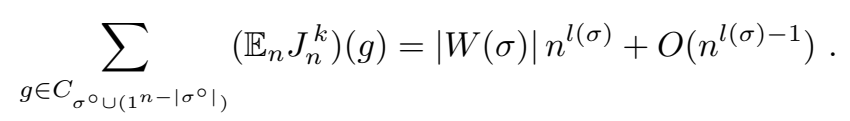

LEMma 2. There is a bijective mapping of $W(\sigma)$ to $N C(\sigma)$ for $\sigma \in \overline{\mathbb{Y}}$.

The proof of Lemma 2 is given in the next subsection.

Combining Eqs. (2) and (3) with Lemma 2, we get

$$
\mathbb{E}_{n} J_{n}^{k}=\sum_{\sigma \in \overline{\mathbb{Y}}_{k}} \sum_{g \in C_{\sigma^{\circ} \cup\left(1^{\left.n-\left|\sigma^{\circ}\right|\right)}\right.}}|N C(\sigma)| n^{l(\sigma)}\left(1+O\left(n^{-1}\right)\right) g .
$$

Since $J_{n}$ commutes with $S(n)$ (= the stabilizer of $*$ in $S(n+1)$ ), so does $\mathbb{E}_{n} J_{n}^{k}$. In other words, $\mathbb{E}_{n} J_{n}^{k}$ is a linear combination of adjacency operators. Note that the correspondence between $\sigma$ and $\sigma^{\circ}$ is one-to-one if $k$ is given. Hence Eq. (4) agrees with Eq. (1). This completes the proof of Theorem 1 . 


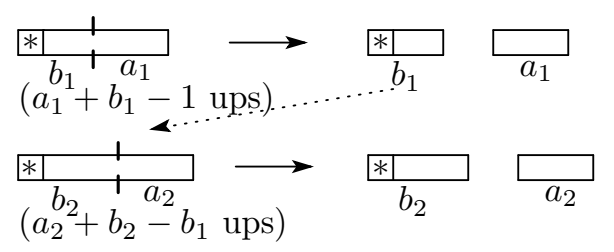

Fig. 3. The first and second down steps

2.2. Proof of Lemma 1. Assume that (i) holds. Let $u$ and $d$ denote the numbers of ups and downs respectively of the $k$-walk from $\varnothing$ to $\rho$ on $\bar{Y}$ associated with the path $e \rightarrow\left(i_{1} *\right) \rightarrow \cdots \rightarrow\left(i_{k} *\right) \cdots\left(i_{1} *\right)$ on $S(n+1)$. We have $u+d=k$ and $u-d=l(\rho)$, hence

$$
u=\frac{k+l(\rho)}{2} \quad \text { and } \quad d=\frac{k-l(\rho)}{2} .
$$

This implies that $k$ and $l(\rho)$ have the same parity. Since a cycle in $\left(i_{k} *\right) \cdots\left(i_{1} *\right)$ which does not contain $*$ cannot be produced until a down step occurs, we have

$$
\operatorname{row}(\rho) \leq d=\frac{k-l(\rho)}{2} \quad \text { hence } \quad k \geq l(\rho)+2 \operatorname{row}(\rho)=|\rho|+\operatorname{row}(\rho) .
$$

Conversely assume that (ii) holds. We can take $\left(i_{k} *\right) \cdots\left(i_{1} *\right) \in S(n)$ of $\rho$-type as follows. Let $\rho=\left(\rho_{1} \geq \rho_{2} \geq \cdots \geq \rho_{\text {row }(\rho)}\right)$. First take distinct $i_{1}, \ldots, i_{\rho_{1}} \in\{1, \ldots, n\}$ and $i_{\rho_{1}+1}=i_{1}$. Then $\left(i_{\rho_{1}+1} *\right)\left(i_{\rho_{1}} *\right) \cdots\left(i_{1} *\right)=\left(i_{1} i_{2} \ldots i_{\rho_{1}}\right) \in S(n)$. Next take distinct $j_{1}, \ldots, j_{\rho_{2}} \in\{1, \ldots, n\} \backslash\left\{i_{1}, \ldots, i_{\rho_{1}}\right\}$ and make $\rho_{2}$-cycle in $S(n)$ similarly. Repeating this procedure to get distinct $\operatorname{row}(\rho)$ cycles in $S(n)$, we count the number of steps as $\left(\rho_{1}+1\right)+\cdots+\left(\rho_{\text {row }(\rho)}+1\right)=|\rho|+\operatorname{row}(\rho) \leq k$. As for the remaining $k-(|\rho|+\operatorname{row}(\rho))$ steps, which is even, we repeat multiplying $(s *)$ by using letter $s$ which never appeared. We have thus (i) $\Leftrightarrow$ (ii). It is obvious that (ii) $\Leftrightarrow$ (iii) holds from the definition of $\sigma^{\circ}$. This completes the proof of Lemma 1.

Proof of Lemma 2. We construct a map $W(\sigma) \rightarrow N C(\sigma)$ for given $\sigma \in \overline{\mathbb{Y}}_{k}$ as follows. Let us assign $\circ$ and $\bullet$ to an up and down step respectively and arrange them according to the given $k$-walk $w \in W(\sigma)$ (e.g. Figure 4 ). We specify the row which contains $*$ for every diagram on $w$. Suppose that the row containing $*$ is divided as Figure 3 at the first down step, then we join the first down vertex $\bullet$ with the $a_{1}(\geq 1)$ left adjacent up vertices $\circ$ to get a block of length $a_{1}+1$. Similarly suppose that the second down step looks like Figure 3, we join the second $\bullet$ with the $a_{2}$ left adjacent o's, skipping the ones contained in the already finished block, to get a block of length $a_{2}+1$. In order to continue this operation until all o's and •'s are joined properly, it suffices to verify that

(i) at each down step the left adjacent o's are not lacking for $a_{i}$

(ii) at the final down step the remaining o's are all joined.

The row finished by a down step may be of length 1 though the row is then not written in $\sigma^{\circ}$. In division into two rows at a down step, the row which does not contain $*$ keeps invariant afterward because we can neglect lower orders. Consider the moment that the $j$ th down step has occurred for $j=1, \ldots, q$ where $q$ is the number of down vertices. The number of vertices which have already appeared is $a_{1}+\cdots+a_{j}+b_{j}+(j-1)$, while the 


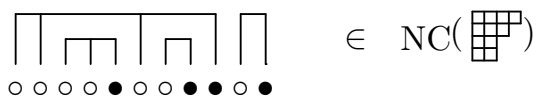

Fig. 4. Example; 11-walk of 7 ups and 4 downs

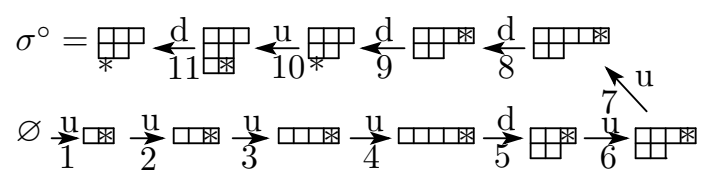

Fig. 5. Example; 11-walk of 7 ups and 4 downs

number of vertices which should be joined is $a_{1}+\cdots+a_{j}+j$. Since $b_{j}-1 \geq 0$ for any $j$, (i) holds. Moreover, since $b_{q}=1$ at the final step, (ii) holds. The resulting partition of $\{1, \ldots, k\}$ is clearly noncrossing. We thus obtain a map $W(\sigma) \rightarrow N C(\sigma)$.

Conversely let $\pi \in N C(\sigma)$ be given. We assign $\bullet$ to the right end vertex of each block and $\circ$ to the other vertices (Figure 4 ). We specify $a_{1}, a_{2}, \ldots$ and then trace backward the above procedure to obtain a $k$-walk in $W(\sigma)$ starting from $\sigma^{\circ}$. (An example is given in Figure 4 and Figure 5.) This completes the proof of Lemma 2.

\section{Concentration phenomenon in irreducible decomposition}

3.1. Fundamental scheme of concentration phenomenon. We summarize a scheme of the concentration phenomenon due to Biane [1], [2] as follows. Let $\chi^{(n)}$ be a positive-definite central function on $S(n)$ and $\tilde{\chi}^{(n)}=\chi^{(n)} / \chi^{(n)}(e)$ its normalization. When we consider the concentration in irreducible decomposition of representation $\pi_{n}$ of $S(n)$, we take $\chi^{(n)}=\chi^{\pi_{n}}$ as the character of $\pi_{n}$. Since $\tilde{\chi}^{(n)}$ is expressed as a convex combination of the normalized irreducible characters of $S(n)$, we have probability $P^{(n)}$ on $\mathbb{Y}_{n}$ determined by

$$
\tilde{\chi}^{(n)}=\sum_{\lambda \in \mathbb{Y}_{n}} P^{(n)}(\lambda) \tilde{\chi}^{\lambda}
$$

The transition measure of $\chi^{(n)}$ is also defined through Eq. (6) as

$$
\mathfrak{m}_{\chi^{(n)}}=\sum_{\lambda \in \mathbb{Y}_{n}} P^{(n)}(\lambda) \mathfrak{m}_{\lambda} .
$$

Taking the regime of $1 / \sqrt{n}$-scaling into account, we set the following assumptions for a growing family $\left\{\chi^{(n)}\right\}$.

Assumption 1. For any $k \in \mathbb{N}$, there exists $m_{k} \in \mathbb{R}$ such that

$$
M_{k}\left(\mathfrak{m}_{\chi^{(n)}}\right) \sim m_{k} n^{k / 2} \quad(n \rightarrow \infty) .
$$

Moreover, the moment problem for the sequence $\left\{m_{k}\right\}_{k \in \mathbb{N}}$ has a unique solution.

Assumption 2. For any $\rho, \sigma, \tau \in \overline{\mathbb{Y}}, \chi^{(n)}$ satisfies:

2a) (decay order)

$$
\tilde{\chi}_{\rho \cup\left(1^{n-|\rho|}\right)}^{(n)}=O\left(n^{-l(\rho) / 2}\right) \quad(n \rightarrow \infty)
$$


2b) (asymptotic factorization)

$$
\tilde{\chi}_{\sigma \cup \tau \cup\left(1^{n-|\sigma|-|\tau|}\right)}^{(n)}-\tilde{\chi}_{\sigma \cup\left(1^{n-|\sigma|}\right)}^{(n)} \tilde{\chi}_{\tau \cup\left(1^{n-|\tau|}\right)}^{(n)}=o\left(n^{-(l(\sigma)+l(\tau)) / 2}\right) \quad(n \rightarrow \infty) .
$$

We can see that for irreducible characters Eq. (8) implies Eq. (9) with a better error $O\left(n^{-(l(\sigma)+l(\tau)) / 2-1}\right)$ by using multiplicativity on the center and an elementary argument on conjugacy classes (see e.g. $§ 3.3$ of [5]). Let $\Delta$ denote the continuous diagram determined from Assumption 1 by

$$
m_{k}=\int_{-\infty}^{\infty} x^{k} \mathfrak{m}_{\Delta}(d x) \quad(k \in \mathbb{N}) .
$$

As Biane showed in [2], Assumptions 1 and 2 yield the concentration at $\Delta \in \mathbb{D}$ in the regime of $1 / \sqrt{n}$-scaling as $n \rightarrow \infty$. More precisely, for arbitrarily given errors $\epsilon_{1}, \epsilon_{2}>0$, we have

$$
P^{(n)}\left(\left\{\lambda \in \mathbb{Y}_{n}|| M_{k}\left(\mathfrak{m}_{\lambda \sqrt{n}}\right)-M_{k}\left(\mathfrak{m}_{\Delta}\right) \mid \geq \epsilon_{1}\right\}\right) \leq \epsilon_{2}
$$

for sufficiently large $n$ (see Eq. (19) for $\lambda^{\sqrt{n}}$ ). If we are dealing with $A$-balanced diagrams for some fixed $A>0$, Eq. (11) implies the concentration at $\Delta$ also in the uniform topology on the continuous diagrams.

A self-contained derivation of the result in this subsection from Theorem 1 is presented in [5]. We state here its outline for the sake of convenience. Since Eq. (22) yields $\tilde{\chi}^{(n)}\left(\mathbb{E}_{n} J_{n}^{k}\right)=M_{k}\left(\mathfrak{m}_{\chi^{(n)}}\right)$ for any $k \in \mathbb{N}$, Assumption 1 (Eq. (7)) is written as

$$
\tilde{\chi}^{(n)}\left(\mathbb{E}_{n} J_{n}^{k}\right) \sim M_{k}\left(\mathfrak{m}_{\Delta}\right) n^{k / 2} \quad(n \rightarrow \infty)
$$

under Eq. (10). A central limit argument based on Theorem 1 contributes to verification of Eq. (12) (hence Assumption 1) for relevant representations. Note that multiplicativity

$$
\tilde{\chi}^{\lambda}\left(\mathbb{E}_{n} J_{n}^{k} \mathbb{E}_{n} J_{n}^{l}\right)=\tilde{\chi}^{\lambda}\left(\mathbb{E}_{n} J_{n}^{k}\right) \tilde{\chi}^{\lambda}\left(\mathbb{E}_{n} J_{n}^{l}\right) \quad\left(\lambda \in \mathbb{Y}_{n} ; k, l \in \mathbb{N}\right)
$$

holds since $\mathbb{E}_{n} J_{n}^{k}$ belongs to the center of $\mathbb{C}[S(n)]$. Applying Theorem 1 , we can show that an asymptotic factorization property

$$
\tilde{\chi}^{(n)}\left(\mathbb{E}_{n} J_{n}^{k} \mathbb{E}_{n} J_{n}^{l}\right)-\tilde{\chi}^{(n)}\left(\mathbb{E}_{n} J_{n}^{k}\right) \tilde{\chi}^{(n)}\left(\mathbb{E}_{n} J_{n}^{l}\right)=o\left(n^{(k+l) / 2}\right) \quad(n \rightarrow \infty)
$$

holds if $\chi^{(n)}$ satisfies Assumption 2. Eqs. (12) and (13) imply that the 'mean' and 'standard deviation' have the asymptotics

$$
\begin{aligned}
& \tilde{\chi}^{(n)}\left(\mathbb{E}_{n} J_{n}^{k}\right) \asymp n^{k / 2}, \\
& \left\{\tilde{\chi}^{(n)}\left(\mathbb{E}_{n} J_{n}^{k} \mathbb{E}_{n} J_{n}^{k}\right)-\tilde{\chi}^{(n)}\left(\mathbb{E}_{n} J_{n}^{k}\right)^{2}\right\}^{1 / 2}=o\left(n^{k / 2}\right),
\end{aligned}
$$

respectively for each $k \in \mathbb{N}$. Then, through a standard discussion relying on the Chebychev inequality, we derive Eq. (11) by using Eq. (22) again.

3.2. Examples. Let us consider irreducible decomposition of a representation of the symmetric group and assume that the characters are computable in an appropriate sense. Through the discussion in $\S 3.1$, we can understand a concentration phenomenon for the representation by applying Theorem 1 in the framework due to Biane. We briefly discuss the following two cases which include several interesting examples.

1. Induction of an irreducible representation of a subgroup.

2. Restriction of a factor representation of the infinite symmetric group $S(\infty)$. 
3.2.1. Let us take Gel'fand pair $(S(m+n), S(m) \times S(n))$ as an example. Here $S(m)$ and $S(n)$ are regarded as the stabilizers in $S(m+n)$ of $m+1, \ldots, m+n$ and $1, \ldots, m$ respectively. For $\lambda \in \mathbb{Y}_{m}$ and $\mu \in \mathbb{Y}_{n}$, consider irreducible decomposition of their outer product

$$
U_{\lambda} \circ U_{\mu}=\operatorname{Ind}_{S(m) \times S(n)}^{S(m+n)} U_{\lambda} \otimes U_{\mu} \simeq \bigoplus_{\nu \in \mathbb{Y}_{m+n}} c_{\lambda \mu}^{\nu} U_{\nu}
$$

$c_{\lambda \mu}^{\nu}$ is a Littlewood-Richardson coefficient. Probability $\mathfrak{L}_{\lambda, \mu}$ on $\mathbb{Y}_{m+n}$ defined by

$$
\mathfrak{L}_{\lambda, \mu}(\nu)=\frac{c_{\lambda \mu}^{\nu} \operatorname{dim} \nu}{\operatorname{dim} \lambda \circ \mu} \quad\left(\nu \in \mathbb{Y}_{m+n}\right)
$$

gives decomposition of the normalized character $\tilde{\chi}^{\lambda \circ \mu}$. We call it the Littlewood-Richardson measure. If we apply the induced character formula and Biane's asymptotic character formula Eq. (26) for balanced Young diagrams, verification of Assumptions 1 and 2 is not difficult. Computing $\tilde{\chi}^{\lambda \circ \mu}\left(\mathbb{E}_{m+n} J_{m+n}^{k}\right)$ by using Theorem 1 , we get the expression of the free cumulant-moment formula for free convolution $\mathfrak{m}_{\lambda} \boxplus \mathfrak{m}_{\mu}$. Thus, as anticipated in Introduction, the concentration phenomenon with respect to the Littlewood-Richardson measure due to Biane [1] is shown within the framework of representations of the symmetric groups.

Let us take Gel'fand pair $(S(2 n), H(n))$ as another example, where $H(n)=\left(\mathbb{Z}_{2}\right)^{n} \rtimes$ $S(n)$. Here we divide $\{1, \ldots, 2 n\}$ into pairs as $\{1, \overline{1}, \ldots, n, \bar{n}\}=\{\mathbf{1}, \ldots, \boldsymbol{n}\}, \boldsymbol{i}=\{i, \bar{i}\}$. $\left(\mathbb{Z}_{2}\right)^{n}$ is regarded as a subgroup of $S(2 n)$ by acting as flips of $i$ and $\bar{i}$, while $S(n)$ by acting as permutations of $\{\mathbf{1}, \ldots, \boldsymbol{n}\}$. Spherical functions on $(S(2 n), H(n))$ are known as zonal polynomials. From a general construction of irreducible representations of a semi-direct product group, the irreducible representations of $H(n)$ are parametrized by $\left\{(m, \lambda, \mu) \mid m \in\{0, \ldots, n\}, \lambda \in \mathbb{Y}_{m}, \mu \in \mathbb{Y}_{n-m}\right\}$. Consider the probability coming from irreducible decomposition of $\operatorname{Ind}_{H(n)}^{S(2 n)} U_{(m, \lambda, \mu)}$ as above. In this case also, Assumptions 1 and 2 are verified through computation by using the induced character formula. As a result, we observe concentration at the limit shape $\Omega$ (Eq. (18)). Restricted on balanced Young diagrams, the concentration is valid in the uniform topology on the continuous diagrams. See [6] for some details in this paragraph.

3.2.2. This second case is pointed out by Biane [2]. As shown by Thoma [16], the factor representations of finite type of $S(\infty)$ are parametrized by

$$
\left\{\alpha=\left(\alpha_{1} \geq \alpha_{2} \geq \cdots \geq 0\right), \beta=\left(\beta_{1} \geq \beta_{2} \geq \cdots \geq 0\right) \mid \sum_{i=1}^{\infty}\left(\alpha_{i}+\beta_{i}\right) \leq 1\right\} .
$$

The value of the character at the conjugacy class corresponding to $\rho \in \mathbb{Y}$ is given by

$$
\psi_{\rho}^{\alpha, \beta}=\prod_{k \geq 2}\left(\sum_{i=1}^{\infty} \alpha_{i}^{k}-\sum_{i=1}^{\infty}\left(-\beta_{i}\right)^{k}\right)^{m_{k}(\rho)} .
$$

Vershik-Kerov [20] constructed realization $U^{\alpha, \beta}$ of the factor representation corresponding to this $\psi^{\alpha, \beta}$. In particular, it is isomorphic to the regular representation if $\alpha=$ $\beta=\mathbf{0}$, while it is the canonical representation on a tensor product of $\mathbb{C}^{N}$ if $\alpha=$ $(1 / N, \cdots, 1 / N, 0, \cdots)(1 / N$ repeated $N$ times $)$ and $\beta=\mathbf{0}$. As shown by Vershik-Kerov $[19], \psi^{\alpha, \beta}$ is captured as the limit of irreducible character $\tilde{\chi}^{\lambda^{(n)}}$ along the sequence 

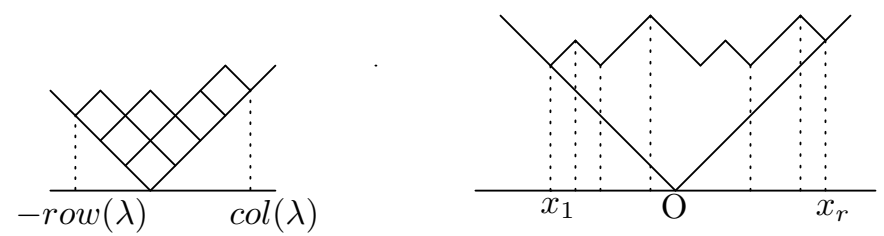

Fig. 6. Young diagram and the min-max coordinates

$\lambda^{(n)} \in \mathbb{Y}_{n}$ such that $\lambda_{i}^{(n)} / n \rightarrow \alpha_{i}$ and $\lambda_{i}^{(n) \prime} / n \rightarrow \beta_{i}$. Here' indicates the transposed Young diagram. Hence, when we discuss concentration phenomenon in irreducible decomposition of $\operatorname{Res}_{S(n)}^{S(\infty)} U^{\alpha^{(n)}, \beta^{(n)}}$ under $1 / \sqrt{n}$-scaling, we should necessarily take sequences $\alpha^{(n)}, \beta^{(n)}$ which converge to 0 pointwise. Since $\tilde{\chi}^{\alpha^{(n)}, \beta^{(n)}}=\left.\psi^{\alpha^{(n)}, \beta^{(n)}}\right|_{S(n)}$ is multiplicative, Assumption 2 is trivial. Assumption 1 is also treated in such a way that the free cumulants of the limit measure are computed by using Eq. (14) and Theorem 1.

\section{Appendices}

4.1. Transition measure and continuous diagram. In order to admit operations of scaling limits, it is convenient to extend the notion of Young diagrams through the expression in a "Russian style" (Figure 6). As references for this subsection, we mention Kerov [9], [10]. See also Ivanov-Olshanski [8]. We restrict ourselves to dealing with centered diagrams. Set

$$
\begin{aligned}
\mathbb{D} & =\left\{\omega: \mathbb{R} \rightarrow \mathbb{R}|| \omega\left(x_{1}\right)-\omega\left(x_{2}\right)|\leq| x_{1}-x_{2}|, \omega(x)=| x \mid \text { for sufficiently large }|x|\right\} \\
& \supset \mathbb{D}_{0}=\left\{\omega \in \mathbb{D} \mid \omega \text { is piecewise linear, } \omega^{\prime}(x)=1 \text { or }-1\right\} \supset \mathbb{Y} .
\end{aligned}
$$

Elements in $\mathbb{D}$ and $\mathbb{D}_{0}$ are called continuous diagrams and rectangular diagrams respectively. A rectangular diagram is determined by the interlacing sequence consisting of positions of its local minima $x_{i}$ and local maxima $y_{i}$ (Figure 6 ):

$$
\lambda \in \mathbb{D}_{0} \leftrightarrow x_{1}<y_{1}<x_{2}<\cdots<x_{r-1}<y_{r-1}<x_{r} \text { such that } \sum_{i=1}^{r} x_{i}=\sum_{i=1}^{r-1} y_{i}
$$

(Note that the min-max coordinates $x_{i}, y_{i}$ of $\lambda \in \mathbb{Y}$ are integers since each box is $\sqrt{2} \times \sqrt{2}$ in the embedding $\mathbb{Y} \subset \mathbb{D}$.) Considering the partial fraction expansion

$$
\frac{\left(z-y_{1}\right) \cdots\left(z-y_{r-1}\right)}{\left(z-x_{1}\right) \cdots\left(z-x_{r}\right)}=\frac{\mu_{1}}{z-x_{1}}+\cdots+\frac{\mu_{r}}{z-x_{r}},
$$

we get atomic probability measure $\sum_{i=1}^{r} \mu_{i} \delta_{x_{i}}$ on $\mathbb{R}$, which has mean 0 since $\sum x_{i}=\sum y_{i}$. Conversely, since $y_{i}$ 's are defined by Eq. (16) if such a probability measure is given, we have a rectangular diagram satisfying (15). The transition measure of $\lambda \in \mathbb{D}_{0}$ is defined as this atomic probability and denoted by $\mathfrak{m}_{\lambda}$. Let $M_{k}(\cdot)$ denote the $k$ th moment of a measure. We have $M_{0}\left(\mathfrak{m}_{\lambda}\right)=1, M_{1}\left(\mathfrak{m}_{\lambda}\right)=0$ and, if $\lambda \in \mathbb{Y}, M_{2}\left(\mathfrak{m}_{\lambda}\right)=|\lambda|$. On the other hand, set $\tau_{\lambda}=\sum_{i=1}^{r} \delta_{x_{i}}-\sum_{i=1}^{r-1} \delta_{y_{i}}$ for $\lambda \in \mathbb{D}_{0}$ satisfying (15). $\tau_{\lambda}$ is called the Rayleigh measure of $\lambda$. Then we see

$$
\sum_{n=0}^{\infty} M_{n}\left(\mathfrak{m}_{\lambda}\right) \frac{1}{z^{n}}=\exp \sum_{k=1}^{\infty} \frac{M_{k}\left(\tau_{\lambda}\right)}{k} \frac{1}{z^{k}}
$$


Especially, $\left\{M_{n}\left(\mathfrak{m}_{\lambda}\right)\right\}_{n \in \mathbb{N}}$ and $\left\{M_{k}\left(\tau_{\lambda}\right)\right\}_{k \in \mathbb{N}}$ are expressed by polynomials of each other. For arbitrary $\omega \in \mathbb{D}$, take an approximating sequence of rectangular diagrams in the uniform topology. By virtue of the moment problem for probabilities and Eq. (17), we obtain uniquely the Rayleigh measure $\tau_{\omega}$ and the transition measure $\mathfrak{m}_{\omega}$. Here $\mathfrak{m}_{\omega}$ and $\tau_{\omega}$ also satisfy Eq. (17). Furthermore, if the support of any $\omega(x)-|x|$ is contained in a fixed compact interval, these correspondences are homeomorphic with respect to the uniform topology on the continuous diagrams and the weak topology on the probabilities. The transition measure and the Rayleigh measure of the limit shape:

$$
\Omega(x)= \begin{cases}\frac{2}{\pi}\left(x \arcsin \frac{x}{2}+\sqrt{4-x^{2}}\right) & (|x| \leq 2), \\ |x| & (|x|>2)\end{cases}
$$

are the standard semi-circle distribution and an arcsine one respectively:

$$
\mathfrak{m}_{\Omega}(d x)=\frac{1}{2 \pi} \sqrt{4-x^{2}} I_{[-2,2]}(x) d x, \quad \tau_{\Omega}(d x)=\frac{1}{\pi \sqrt{4-x^{2}}} I_{(-2,2)}(x) d x .
$$

If we consider a rescaled diagram $\omega^{s}(x)=s^{-1} \omega(s x)$ for $\omega \in \mathbb{D}$ and $s>0$, we have

$$
M_{k}\left(\mathfrak{m}_{\omega^{s}}\right)=\frac{1}{s^{k}} M_{k}\left(\mathfrak{m}_{\omega}\right) \quad(k \in \mathbb{N}) .
$$

4.2. A trace formula. Let $\lambda \in \mathbb{Y}_{n}$ have $x_{1}<y_{1}<\cdots<y_{r-1}<x_{r}$ as the min-max coordinates (Figure 6). Putting a box at the 'valley' of min coordinate $x_{j}$ of $\lambda$, we obtain $r$ diagrams in $\mathbb{Y}_{n+1}$, denoted by $\Lambda_{j}(j=1, \ldots, r)$. By using the hook formula for dimensions of irreducible representations, we can verify that the transition measure of $\lambda$ is given by

$$
\mathfrak{m}_{\lambda}=\sum_{j=1}^{r} \frac{\operatorname{dim} \Lambda_{j}}{(n+1) \operatorname{dim} \lambda} \delta_{x_{j}}
$$

See [10], Chapter 4, 1.4. The mass $\operatorname{dim} \Lambda /(n+1) \operatorname{dim} \lambda$ agrees with the transition probability from $\lambda$ to $\Lambda$ of the Markov chain on the Young graph $\mathbb{Y}$ which is called the Plancherel growth process. On the other hand, by using well-known spectral structure of the Jucys-Murphy elements (see e.g. [15]), we see

$$
\tilde{\chi}^{\lambda}\left(\mathbb{E}_{n} J_{n}^{k}\right)=\sum_{j=1}^{r} x_{j}^{k} \frac{\operatorname{dim} \Lambda_{j}}{(n+1) \operatorname{dim} \lambda} .
$$

Eqs. (20) and (21) yield the following trace formula (see [3]):

$$
\tilde{\chi}^{\lambda}\left(\mathbb{E}_{n} J_{n}^{k}\right)=M_{k}\left(\mathfrak{m}_{\lambda}\right) \quad\left(k \in \mathbb{N}, \lambda \in \mathbb{Y}_{n}\right)
$$

4.3. Polynomial functions on Young diagrams, the conjugacy class algebra. We review these materials as a background of such an expression as Eq. (1). We refer to KerovOlshanski [11], Ivanov-Olshanski [8], Ivanov-Kerov [7] and Kerov [10] for details. Recall $\left\{M_{k}\left(\tau_{\lambda}\right)\right\}_{k \in \mathbb{N}}$ and $\left\{M_{k}\left(\mathfrak{m}_{\lambda}\right)\right\}_{k \in \mathbb{N}}$ are related to each other by polynomials in view of Eq. (17). They are expressed as polynomials in the min-max coordinates of $\lambda$. We consider the weight degree by regarding $M_{k}\left(\tau_{\lambda}\right)$ as homogeneous with degree $k$. Set

$$
\Sigma_{\rho}(\lambda)= \begin{cases}|\lambda| \frac{|\rho|}{-} \tilde{\chi}_{\rho \cup(1|\lambda|-|\rho|)}^{\lambda} & (|\lambda| \geq|\rho|) \\ 0 & (|\lambda|<|\rho|)\end{cases}
$$




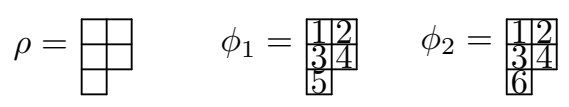

Fig. 7. $g_{\phi_{1}}=g_{\phi_{2}}=(12)(34) \in S(6)$

for $\rho \in \mathbb{Y}$, and $\Sigma_{k}(\lambda)=\Sigma_{(k)}(\lambda)$ for $k \in \mathbb{N}$. Applying the Frobenius formula for the value of an irreducible character at a cycle, we can get

$$
\Sigma_{k}(\lambda)=-\frac{1}{k}\left[z^{-1}\right]\left\{\frac{1}{G_{\mathfrak{m}_{\lambda}}(z)} \cdots \frac{1}{G_{\mathfrak{m}_{\lambda}}(z-k+1)}\right\}
$$

where $G_{\mu}(z)$ denotes the Cauchy transform of the measure $\mu$. On the other hand, the Voiculescu formula for the $R$-transform gives the expression for free cumulants:

$$
R_{k+1}\left(\mathfrak{m}_{\lambda}\right)=-\frac{1}{k}\left[z^{-1}\right]\left\{\left(\frac{1}{G_{\mathfrak{m}_{\lambda}}(z)}\right)^{k}\right\} .
$$

Comparing Eq. (23) and Eq. (24), we deduce

$$
\Sigma_{k}(\lambda)=K_{k}\left(R_{2}\left(\mathfrak{m}_{\lambda}\right), \cdots, R_{k+1}\left(\mathfrak{m}_{\lambda}\right)\right)=R_{k+1}\left(\mathfrak{m}_{\lambda}\right)+\cdots
$$

where the lower terms $(\cdots)$ consist of a polynomial in $R_{2}, \cdots, R_{k-1}$, each term having the weight degree of the same parity with $k+1$. $K_{k}$ is called a Kerov polynomial. See Biane [3]. Consequently, $\left\{M_{k}\left(\tau_{\lambda}\right)\right\},\left\{M_{k}\left(\mathfrak{m}_{\lambda}\right)\right\},\left\{R_{k}\left(\tau_{\lambda}\right)\right\},\left\{R_{k}\left(\mathfrak{m}_{\lambda}\right)\right\}$ and $\left\{\Sigma_{k}(\lambda)\right\}$ generate the same algebra $\mathfrak{A}$. We call $\mathfrak{A}$ the polynomial functions on $\mathbb{Y}$ after Kerov-Olshanski [11]. Note that the weight degree of $\Sigma_{k}$ is $k+1$.

The structure of $\mathfrak{A}$ can be transformed into the conjugacy class algebra. Set

$$
\Phi_{\rho ; n}=\left\{\phi:\{b\}_{b \in \rho} \rightarrow\{1,2, \ldots, n\} \mid \text { one-to-one map }\right\}
$$

for $\rho \in \mathbb{Y}$ and $n \geq|\rho|$. Let $g_{\phi}$ be the element in $S(n)$ with cycle structure determined by $\phi$. For example, see Figure 7. Set

$$
a_{\rho ; n}= \begin{cases}\sum_{\phi \in \Phi_{\rho ; n}} g_{\phi} & (|\rho| \leq n) \\ 0 & (|\rho|>n)\end{cases}
$$

and $a_{k ; n}=a_{(k) ; n}$ for $k \in \mathbb{N}$. We see

$$
a_{\rho ; n}=n \frac{|\rho|}{\frac{A_{\rho \cup\left(1^{n-|\rho|}\right)}}{\left|C_{\rho \cup\left(1^{n-|\rho|}\right)}\right|}} \quad(|\rho| \leq n) .
$$

It is remarkable that structure constants of products of $a_{\rho ; n}$ are independent of $n$ :

$$
a_{\sigma ; n} a_{\tau ; n}=\sum_{\rho} f_{\sigma, \tau}^{\rho} a_{\rho ; n} \quad\left(f_{\sigma, \tau}^{\rho} \in \mathbb{Z}\right)
$$

which was shown by Ivanov-Kerov [7]. Clearly $\tilde{\chi}^{\lambda}\left(a_{\rho ; n}\right)=\Sigma_{\rho}(\lambda)$ holds for $\lambda \in \mathbb{Y}_{n} \cdot \tilde{\chi}^{\lambda}$ is multiplicative on the center of $\mathbb{C}[S(n)]$. By virtue of Eq. (25), the correspondence $a_{k ; n} \longleftrightarrow \Sigma_{k}$ (given by taking the irreducible characters) defines the weight degree in the conjugacy class algebra consistently (independently of $n$ ). Eq. (22) indicates that $\mathbb{E}_{n} J_{n}{ }^{k}$ corresponds to $M_{k}(\mathfrak{m}$.) and has weight degree $k$.

Kerov polynomials yield the following asymptotic character formula of Biane [1]:

$$
\tilde{\chi}_{\rho \cup\left(1^{n-|\rho|}\right)}^{\lambda}=n^{-|\rho|} \prod_{j \geq 2} R_{j+1}\left(\mathfrak{m}_{\lambda}\right)^{m_{j}(\rho)}+O\left(n^{-l(\rho) / 2-1}\right) \quad(n \rightarrow \infty)
$$


for $\rho \in \overline{\mathbb{Y}}$ and $\lambda \in \mathbb{Y}_{n}(A)$ where $\mathbb{Y}_{n}(A)=\left\{\lambda \in \mathbb{Y}_{n} \mid \operatorname{row}(\lambda), \operatorname{col}(\lambda) \leq A \sqrt{n}\right\}$ for some $A>0$ (so-called $A$-balanced Young diagrams). In fact, Ivanov-Olshanski proved Eq. (26) in [8] along this line. See [5] for derivation of Eq. (26) from Theorem 1.

\section{References}

[1] P. Biane, Representations of symmetric groups and free probability, Adv. Math. 138 (1998), 126-181.

[2] P. Biane, Approximate factorization and concentration for characters of symmetric groups, Internat. Math. Res. Notices 2001, no. 4, 179-192.

[3] P. Biane, Characters of symmetric groups and free cumulants, in: [17], 185-200.

[4] A. Hora, A noncommutative version of Kerov's Gaussian limit for the Plancherel measure of the symmetric group, in: [17], 77-88.

[5] A. Hora, Remark on Biane's character formula and concentration phenomenon in asymptotic representation theory, submitted, 2004.

[6] A. Hora, Jucys-Murphy elements, walks on Young graph and applications to asymptotic representation theory, unpublished, 2004.

[7] V. Ivanov and S. Kerov, The algebra of conjugacy classes in symmetric groups and partial permutations, J. Math. Sci. 107 (2001), 4212-4230.

[8] V. Ivanov and G. Olshanski, Kerov's central limit theorem for the Plancherel measure on Young diagrams, in: Symmetric Functions 2001, S. Fomin (ed.), Kluwer Academic Publishers, 2002, 93-151.

[9] S. V. Kerov, Transition probabilities for continual Young diagrams and the Markov moment problem, Funct. Anal. Appl. 27 (1993), 104-117.

[10] S. V. Kerov, Asymptotic Representation Theory of the Symmetric Group and its Applications in Analysis, MMONO 219, Amer. Math. Soc., 2003.

[11] S. Kerov and G. Olshanski, Polynomial functions on the set of Young diagrams, C. R. Acad. Sci. Paris 319 (1994), Série I, 121-126.

[12] B. F. Logan and L. A. Shepp, A variational problem for random Young tableaux, Adv. Math. 26 (1977), 206-222.

[13] P. Śniady, Free probability and representations of large symmetric groups, math. CO/0304275, 2003.

[14] P. Sniady, Asymptotics of characters of symmetric groups, Gaussian fluctuations of Young diagrams and genus expansion, math. CO/041164, 2004.

[15] I. Terada and K. Harada, Group Theory, Iwanami-Shoten, 1997 (in Japanese).

[16] E. Thoma, Die unzerlegbaren, positiv-definiten Klassenfunktionen der abzählbar unendlichen, symmetrischen Gruppe, Math. Z. 85 (1964), 40-61.

[17] A. M. Vershik (ed.), Asymptotic Combinatorics with Applications to Mathematical Physics, Lect. Notes in Math. 1815, Springer, 2003.

[18] A. M. Vershik and S. V. Kerov, Asymptotics of the Plancherel measure of the symmetric group and the limiting form of Young tableaux, Soviet Math. Dokl. 18 (1977), 527-531.

[19] A. M. Vershik and S. V. Kerov, Asymptotic theory of characters of the symmetric group, Funct. Anal. Appl. 15 (1981), 246-255.

[20] A. M. Vershik and S. V. Kerov, Characters and factor representations of the infinite symmetric group, Soviet Math. Dokl. 23 (1981), 389-392. 
